INPLASY

PROTOCOL

To cite: Xiao et al. Abrocitinib for the treatment of moderateto-severe atopic dermatitis: a systematic review and metaanalysis of randomized controlled trials. Inplasy protocol 202160083. doi: 10.37766/inplasy2021.6.0083

Published: 23 June 2021

Corresponding author: Jie Li

xylijie@csu.edu.cn

Author Affiliation:

Xiangya Hospital

Support: NSFC.

Review Stage at time of this submission: Formal screening of search results against eligibility criteria.

Conflicts of interest:

None declared.

\section{Abrocitinib for the treatment of moderate-to-severe atopic dermatitis: a systematic review and meta-analysis of randomized controlled trials}

Xiao, YJ1; Yuan, Y2; Hong, ZY3; Li, J4.

Review question / Objective: What is the clinical efficacy and safety of treatment with abrocitinib in moderate to severe atopic dermatitis?

Condition being studied: Atopic dermatitis. Efficacy and safety of abrocitinib for atopic dermatitis.

Eligibility criteria: Inclusion: (1) patients given a diagnosis of $A D$ and treated with abrocitinib; (2) double-blind, randomized, placebo-controlled studies; (3) outcome measures including EASI, IGA, PP-NRS score and DLQI, as well as AEs. Exclusion: Duplicate publications, nonhuman studies and studies that did not report on prespecified outcomes.

INPLASY registration number: This protocol was registered with the International Platform of Registered Systematic Review and Meta-Analysis Protocols (INPLASY) on 23 June 2021 and was last updated on 23 June 2021 (registration number INPLASY202160083).

\section{INTRODUCTION}

Review question / Objective: What is the clinical efficacy and safety of treatment with abrocitinib in moderate to severe atopic dermatitis?

Condition being studied: Atopic dermatitis. Efficacy and safety of abrocitinib for atopic dermatitis.

\section{METHODS}

Search strategy: We used the search terms "abrocitinib" or "PF-04965842" and "atopic dermatitis" or "atopic eczema" and searched the following databases: i) MEDLINE (via PubMed); ii) Cochrane Controlled Trials Register; iii) EMBASE, and; iiii) Web of Science. In addition, we searched trials registered on ClinicalTrials.gov. We will also review 
references of included studies and previous reviews. Searches were restricted to publications in the English. Unpublished studies will be sought.

Participant or population: Patient with moderate-to-severe atopic dermatitis received treatment of abrocitinib.

Intervention: Abrocitinib, irrespective of dose, frequency and course duration.

Comparator: Placebo and/or usual care/ standard of care.

Study designs to be included: We will include randomised trials to assess the efficacy and safety of abrocitinib for moderate-to-severe atopic dermatitis.

Eligibility criteria: Inclusion: (1) patients given a diagnosis of $A D$ and treated with abrocitinib; (2) double-blind, randomized, placebo-controlled studies; (3) outcome measures including EASI, IGA, PP-NRS score and DLQI, as well as AEs. Exclusion: Duplicate publications, nonhuman studies and studies that did not report on prespecified outcomes.

Information sources: i) MEDLINE (via PubMed) ; ii) Cochrane Controlled Trials Register; iii) EMBASE, and ; iiii) Web of Science. In addition, we searched trials registered on ClinicalTrials.gov.

Main outcome(s): - IGA - EASI score - Peak Pruritus Numerical Rating Scaleworst (PPNRS) - DLQI - Safety (adverse events).

Quality assessment / Risk of bias analysis: We will use the Cochrane Risk of Bias tool for randomised trials to assess the risk of bias of the included studies (Higgins 2011). The assessment includes the following aspects: (1) Random sequence generation (selection bias) (2) Allocation concealment (selection bias) (3) Blinding of participants and personal (performance bias) (4) Blinding of outcomes assessment (detection bias) (5) Incomplete outcome data (attrition bias) (6) Selective reporting (reporting bias).
Strategy of data synthesis: Data suitable to be pooled will be meta-analyzed using Review Manager (RevMan, version 5.3.5., Cochrane Collaboration, Oxford, UK) or STATA software (v.15.0, STATA Corp). Dichotomous data will be calculated by using relative risk (RR) with 95\% confidence intervals (Cls). Continuous data measured using similar scales will be summarized by using mean differences (MD) with $95 \%$ Cls, whilst continuous data measured using different scales will be summarized using standardized mean differences (SMD) with $95 \%$ Cls. Statistical significance will be assumed at $P<0.05$. Heterogeneity will be assessed using the $I^{2}$ statistic. If there is found to be no substantial heterogeneity, we will calculate the pooled estimates of the treatment effect using a fixed effect model, otherwise, a random effects analysis will be carried out if substantial heterogeneity is observed $\left(I^{2}>50 \%\right.$ or $\left.\mathrm{P}<0.1\right)$. For all meta-analysis with at least 10 included studies, we will assess publication bias by visual inspection of the Begg's funnel plot, and statistically using the Egger's test for small-study effects (funnel plot asymmetry).

Subgroup analysis: We will explore the impact of: different dosage, age, and risk of bias.

Sensitivity analysis: Sensitivity analyses would be used to estimates bias.

Country(ies) involved: China.

Keywords: Abrocitinib; atopic dermatitis; efficacy; safety.

Contributions of each author:

Author 1 - Yangjian Xiao.

Email: 2762616707@qq.com

Author 2 - Yuan Yan.

Email: yy12267708@163.com

Author 3 - Zhiyu Hong.

Email: 2201724485@qq.com

Author 4 - Jie Li.

Email: xylijie@csu.edu.cn 